\title{
Distrator ósseo transpalatal como opção para expansão maxilar: Relato de caso
}

\author{
Transpalatal bone distractor as an option for maxillary expansion: Case report \\ Distractor óseo transpalatino como opción para la expansión maxilar: Reporte de caso
}

\author{
Kim Henderson Carmo Ribeiro \\ ORCID: https://orcid.org/0000-0003-3325-5897 \\ Universidade Estadual Paulista, Brasil \\ E-mail: kimhenderson@hotmail.fr \\ Ana Julia Moreno Barreto \\ ORCID: https://orcid.org/0000-0002-0067-1192 \\ Universidade Estadual Paulista, Brasil \\ E-mail: anajuliamorenobarreto1@hotmail.com \\ Ana Paula Simões Corrêa \\ ORCID: https://orcid.org/0000-0002-8207-4606 \\ Universidade Estadual Paulista, Brasil \\ E-mail: paulinha_odonto@hotmail.com \\ Mariza Akemi Matsumoto \\ ORCID: https://orcid.org/0000-0001-5389-0105 \\ Universidade Estadual Paulista, Brasil \\ E-mail:mariza.am@gmail.com \\ Waldemar Pereira Junior \\ ORCID: https://orcid.org/0000-0003-4385-1190 \\ Faculdade de Odontologia da APCD, Brasil \\ E-mail: jrwp1969@gmail.com \\ Ângela Alves de Aguiar Goto \\ ORCID: https://orcid.org/0000-0003-2598-3595 \\ Faculdade Ibeco, Brasil \\ E-mail: clinicafacial@hotmail.com
}

\begin{abstract}
Resumo
A discrepância transversa da maxila é comumente observada em pacientes que procuram tratamento ortodôntico, para uma adequada oclusão. É frequentemente definida como uma mordida cruzada posterior e apinhamento dentário anterior. Várias abordagens estão disponíveis para tratar esta discrepância e alcançar a expansão maxilar, sendo conseguida através de técnicas, cirúrgicas e não cirúrgicas. A necessidade de se utilizar a técnica cirúrgica advém quando o paciente já atingiu a maturidade esquelética, desta forma é possível alcançar a dimensão correta da maxila por meio de aparelhos dento-muco-suportados, dento-suportados e ósseo-suportados. No presente caso, a utilização de um aparelho ósseo-suportado mostra vantagens clínicas significativas sobre as outras técnicas uma vez que não observa-se danos aos tecidos ou elementos dentais e sim efeitos exclusivos aos tecidos ósseos resultando em um aumento significativo do arco maxilar e melhora da oclusão, além de melhora respiratória.
\end{abstract}

Palavras-chave: Técnica de expansão palatina; Má oclusão; Arcada ósseodentária; Técnicas de movimentação dentária; Ortodontia.

\begin{abstract}
Transverse maxillary discrepancy is commonly observed in patients seeking orthodontic treatment for adequate occlusion. Is defined with a posterior crossbite and anterior crowding. Several devices are available to address this discrepancy and achieve maxillary expansion, that can be achieved through surgical and non-surgical techniques. Surgical procedure is indicated when the patient has reached skeletal maturity, in this way it is possible to reach the correct size of the maxilla using dento-mucus-supported, dento-supported and bone-supported devices. In the present case, the use of a bone-supported device shows the advantages over the other techniques since there is no damage to tissues or dental elements showing only effects to bone tissues resulting in a significant increase in the maxillary arch and a better occlusion in addition to respiratory improvement.
\end{abstract}

Keywords: Palatal expansion technique; Malocclusion; Jaw; Tooth movement techniques; Orthodontics.

\section{Resumen}

La discrepancia maxilar transversal se observa comúnmente en pacientes que buscan tratamiento de ortodoncia para una oclusión adecuada. A menudo se define como una mordida cruzada posterior con apiñamiento anterior. Hay varios enfoques disponibles para abordar esta discrepancia y lograr la expansión maxilar, esto se puede lograr 
mediante técnicas, quirúrgicas y no quirúrgicas. La necesidad de utilizar la técnica quirúrgica surge cuando el paciente ha alcanzado la madurez esquelética, por lo que es posible alcanzar el tamaño correcto del maxilar utilizando dispositivos dentomucosos, dentosoportados y óseos. En el presente caso, el uso de un dispositivo óseo presenta importantes ventajas clínicas sobre las otras técnicas, ya que no hay daño a los tejidos ni a los elementos dentales, sino solo efectos a los tejidos óseos que resultan en un aumento significativo del arco maxilar y una mejor oclusión del paciente además de la mejoría respiratoria.

Palabras clave: Técnica de expansión palatina; Maloclusión; Maxilares; Técnicas de movimiento dental; Ortodoncia.

\section{Introdução}

A discrepância transversa da maxila, apresentada como mordida cruzada unilateral ou bilateral posterior e/ou apinhamento, é uma má oclusão comum entre adolescentes e adultos. Dessa forma, aparelhos e técnicas pertinentes para estes casos, foram concebidos com o intuito de expandir estas maxilas (Zhou, et al., 2014). O método de distração osteogênica acerca de atresias maxilares mostra-se efetivo a mais de 300 anos (Sahoo, et al., 2019; Ylikontiola, et al., 2015). Compreendese como distração osteogênica da maxila a aplicação de uma rápida força transversal na dentição superior de modo a interromper a sutura palatina mediana levando a expansão pela remodelação óssea (Carlson, et al., 2016; Lehman \& Haas, 1989). A mesma é conseguida de diversas maneiras, e quando se trata de um paciente adulto com maturação esquelética, há necessidade de uma expansão rápida da maxila assistida cirurgicamente (ERMAC) (Kee-Joon, et al., 2018) sob anestesia geral ou local, onde é realizada uma osteotomia do tipo Le Fort I em conjunto com uma osteotomia palatina (Tehranchi, et al., 2013).

Diversos aparelhos estão disponíveis com o objetivo de se expandir estas arcadas dentárias (Pereira, et al., 2018), dentre os mais utilizados citam-se: aparelhos do tipo Haas (dento-muco-suportado) e Hyrax (dento-suportado) (Araújo, et al., 2020). No entanto, estes aparelhos podem causar alguns efeitos indesejáveis sobre os elementos dentários e tecidos de suporte (Araújo, et al., 2020). Dentre os mais modernos aparelhos, os distratores ósseo-suportados oferecem a vantagem clínica, apresentando menor morbidade e estresse dos elementos dentais pois a transmissão de força é direta do parafuso de expansão para as estruturas esqueléticas, não utilizando de dentes para ancoragem, sendo este aspecto especialmente útil em pacientes com dentições reduzidas ou envolvimento periodontal (Kunz, et al., 2016).

Desta forma, este trabalho tem por objetivo relatar um caso clínico onde utilizou-se distrator transpalatal (TPD) para obter a expansão da maxila de uma paciente com deficiência transversa da maxila.

\section{Metodologia}

O trabalho em questão aborda o caso de uma paciente, de forma descritiva e qualitativa (Pereira, et al., 2018), portadora de uma deficiência transversa de maxila, com o objetivo de detalhar um procedimento de expansão cirúrgica (Tehranchi, et al., 2013), sendo autorizado pelo paciente por meio de um Termo de Consentimento Livre e Esclarecido (TCLE) elaborado em linguagem fácil para obtenção do conhecimento acerca do destino das informações pessoais colhidas além dos riscos e benefícios para tal. Este trabalho foi enviado ao CEP/CONEP pela Plataforma Brasil. Além disso, tem por objetivo oferecer uma breve revisão da literatura, por meio da busca de artigos científicos e estudos de caso nas bases de dados MEDLINE, PubMed e Google Scholar, sobre as vantagens de um distrator ósseo-suportado em relação a outros aparelhos com o mesmo fim, bem como os riscos e benefícios deste tratamento.

\section{Relato do Caso}

Paciente do sexo feminino, caucasiana, 50 anos, compareceu no consultório odontológico com queixa principal de 'mordida errada'. Na avaliação clínica (Figura 1A) e radiográfica (Figura 1B), observou-se ausência de elementos dentários 
posteriores bilateralmente na região mandibular, atresia maxilar e mordida cruzada posterior. $\mathrm{O}$ tratamento proposto para a paciente, afim de se corrigir esta deformidade dento-facial foi a realização de uma ERMAC devido à idade da paciente, onde já ocorreu ossificação da sutura palatina mediana, utilizando um TPD, sob anestesia geral. A paciente foi internada no dia da cirurgia, realizou os exames pré-operatórios, que se encontraram dentro da normalidade e sem relevância nesse relato de caso.

Figura 1. Pré-operatório. A- Paciente na vista frontal sorrindo. B- Radiografia panorâmica pré-operatória.

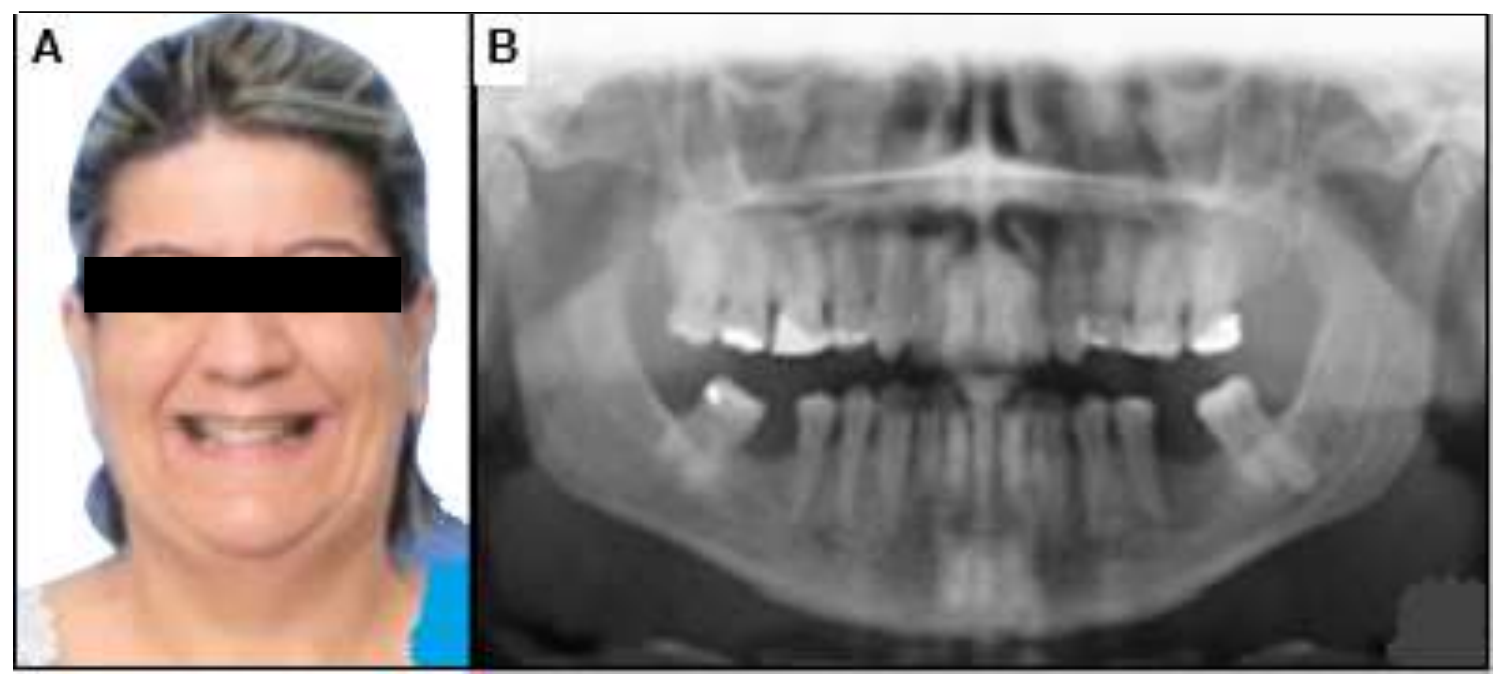

Fonte: Arquivo pessoal.

Sob anestegia geral e intubação naso-traqueal foram realizadas incisões bilaterais na mucosa palatina de forma a expor a superfície óssea e o distrator transpalatal (Slim PRF 9mm E, Traumec ${ }^{\circledR}$ ) foi posicionado transversalmente no palato entre os pré-molares na região mediana do mesmo, com auxílio da pinça posicionadora, mantendo-o afastado da mucosa palatina para adequada higiene e inserção da chave de ativação. Suas bases foram fixadas ao osso no local das incisões com parafusos autoperfurantes de diâmetro e comprimento adequados, posicionando as bases do distrator nos lados direito e esquerdo, conforme gravação presente no produto, "R" e "L" (Figura 2A), respectivamente. Após a instalação do distrator e da realização das osteotomias, do tipo Le Fort I (Figura 2B) com auxílio de uma serra oscilante, a sutura palatina mediana foi esculpida para a divisão do meio palatal e a região do tubérculo também foi mobilizada com um cinzel para a completa desarticulação (Bräutigam, M. et al., 2018). Em seguida, com a chave articulada verificou-se a total separação dos segmentos ósseos. A ativação foi realizada no sentido posterior, promovendo a abertura do distrator até $1 \mathrm{~mm}$ (Figura 2C e 2D), uma expansão mínima, evitando lacerações da mucosa palatina. Uma vez verificada a separação dos segmentos ósseos, foi utilizada a chave de ativação no sentido inverso, de posterior para anterior, retornado o distrator na posição fechada.

A cirurgia ocorreu conforme planejado, sem intercorrências. Após três dias de pós-operatório, a paciente continuou o tratamento com o ortodontista iniciando a ativação do distrator com 1/4 de volta de $12 \mathrm{em} 12$ horas durante 14 dias, abrindo um diastema de $8 \mathrm{~mm}$ entre os incisivos (Figura 2E) e promovendo separação dos ossos palatais observado pela radiografia oclusal (Figura 2E). Continuou-se o tratamento ortodôntico, colagem do aparelho fixo com finalidade de alinhamento e nivelamento dos elementos dentais e uma nova radiografia panorâmica foi solicitada (Figura 2F). O TPD foi mantido por 6 meses na cavidade bucal servindo como contenção e posteriormente removido em âmbito hospitalar sob anestesia geral. Após 2 anos de pós operatório, observa-se estabilização da expansão adquirida, sem recidiva ou efeitos negativos nos tecidos de suporte além de melhora da respiração nasal da paciente. 
Figura 2. Trans-operatório e pós-operatório. A- Exemplo do distrator transpalatal utilizado (Slim PRF 9mm E, Traumec®). BOsteotomia Le Fort 1. C- Ativação do distrator transpalatal e diastema de 1mm entre os incisivos centrais. D- Exemplo do distrator transpalatal aberto. E- Diastema de 8mm após 14 dias de ativação do TPD. F- Radiografia oclusal mostrando separação dos ossos palatais. G- Radiografia panorâmica pós-operatória e presença de aparelhagem fixa inferior.

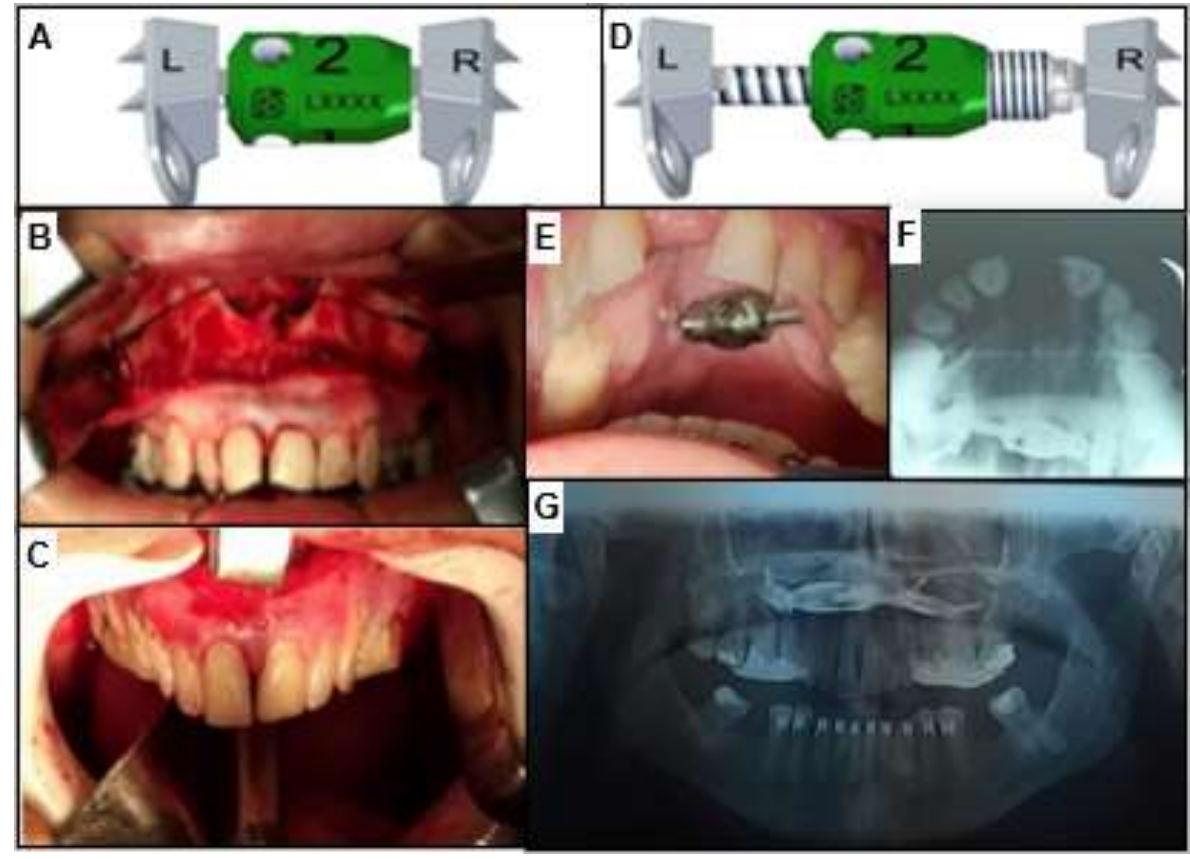

Fonte: Arquivo pessoal.

\section{Discussão}

Uma dimensão transversa da maxila adequada é um componente crítico da oclusão estável e contribui positivamente para a estética do sorriso (Gogna, et al., 2020; Zhou, et al., 2014). A hipoplasia transversa maxilar, em adultos que atingiram a maturação óssea, havendo consolidação da sutura é frequentemente tratada através de expansão cirúrgica das maxilas (Zhou, et al., 2014; Suri \& Taneja 2008). No presente caso, a indicação de uma ERMAC deu-se devido a total consolidação da sutura palatina mediana e necessidade e uma grande expansão da maxila para tratamento dos apinhamentos dentários e correção da mordida cruzada posterior da paciente. O tratamento é uma combinação de procedimentos ortodônticos e cirúrgicos e fornece espaço na arcada dentária para alinhamento dos dentes. Existem três principais tipos de aparelhos expansores e são divididos com relação ao tipo de ancoragem (Pereira, et al., 2018): dento-suportado, dento-muco-suportado e ósseo-suportado. Os dispositivos suportados em dente ou mucosa possuem algumas contra-indicações, como nos casos de pacientes com agenesias ou perdas dentárias, havendo então uma impossibilidade de suporte para este tipo de aparelho, além de ser contra-indicado em casos de paciente com presença de periodontite ou mesmo gengivite (Araújo, et al., 2020). Quando aparelhos suportados em estruturas dentárias são utilizados, os mesmos ocasionam uma expansão da arcada dentária, havendo uma inclinação das raízes em relação ao osso alveolar (Lima, et al., 2011). Isto ocorre devido à movimentação de dentes e não, da estrutura óssea, o que torna o tratamento imprevisível, sendo estes os motivos pela nossa escolha em se utilizar um aparelho totalmente suportado em osso.

Ao utilizar o TPD, a força é aplicada alta na abóbada palatina e a inclinação segmentar no plano coronal é, portanto, mínimo. Como o dispositivo é suportado em tecido ósseo, recidiva dentária durante e após a expansão é evitada, bem como a compressão do ligamento periodontal, reabsorção da raiz vestibular, fenestração e extrusão de dentes não devem ser temidos 
(Neyt, et al., 2002; Kunz \& Linz et al., 2016), uma vez que este dispositivo diminui o stress mecânico sobre os dentes, observado no presente caso em questão.

Ainda que a utilização do TPD indique a necessidade de dois tempos cirúrgicos com a colocação do aparelho e retirada do mesmo, a utilização desse tipo de aparelho protege os dentes por induzir mais alterações ósseas que dentárias, sendo isso uma pré-condição para estabilidade pós-operatória (Santos, et al., 2012) sendo observado em nosso caso com o acompanhamento de dois anos. Além disso, O procedimento também provoca um aumento substancial da base apical maxilar e da abóbada palatina, proporcionando espaço para a língua para a deglutição correta e evitando recaídas. Além disso ocorreu uma melhora subjetiva da respiração nasal associada ao procedimento cirúrgico, relatada pela paciente e apoiado pela literatura como efeito pós-operatório (Di Carlo, et al., 2017; Lagravere, et al., 2006).

Todavia, existem possíveis complicações no ato cirúrgico, como infecção sinusal, comunicação/fístula buco-sinusal, parestesia, fratura indesejada durante as osteotomias, desvio septal durante o ato de expansão entre outras (Koudstaal, et al., 2005), no entanto, todas essas possibilidades são facilmente eliminadas através de um planejamento cirúrgico adequado e pleno domínio da técnica cirúrgica por meio de táticas pertinentes ao caso (Filho, et al., 2015).

\section{Conclusão}

A expansão rápida da maxila assistida cirurgicamente trata-se de um procedimento seguro e eficaz para a correção da deficiência transversa maxilar em pacientes adultos. Um adequado diagnóstico e um plano de tratamento em conjunto pelo cirurgião bucomaxilofacial e ortodontista possibilitam sucesso da correção dessas deficiências. Para tanto infere-se que o uso de dispositivos ósseos suportados, como o distrator transpalatal utilizado no presente caso, são mais adequados para a distração osteogênica da maxila, uma vez que as forças de expansão são aplicadas sobre o osso palatino, evitando movimentações dentárias. Dessa forma, consideramos ser um dispositivo que pode ser usado para realizar a ERMAC mostrando-se eficiente e de fácil instalação quando o cirurgião dentista está familiarizado com a técnica.

\section{Referências}

Araújo, M. C., Bocato, J. R., Oltramari, P. V., de Almeida, M. R., Conti, A. C., \& Fernandes, T. M. (2020). Tomographic evaluation of dentoskeletal effects of rapid maxillary expansion using Haas and Hyrax palatal expanders in children: A randomized clinical trial. Journal of clinical and experimental dentistry, 12(10), e922-e930. https://doi.org/10.4317/jced.57277

Carlson, C., Sung, J., McComb, R. W., Machado, A. W., \& Moon, W. (2016). Microimplant-assisted rapid palatal expansion appliance to orthopedically correct transverse maxillary deficiency in an adult. American journal of orthodontics and dentofacial orthopedics: official publication of the American Association of Orthodontists, its constituent societies, and the American Board of Orthodontics, 149(5), 716-728. https://doi.org/10.1016/j.ajodo.2015.04.043

Di Carlo, G., Saccucci, M., Ierardo, G., Luzzi, V., Occasi, F., Zicari, A. M., Duse, M., \& Polimeni, A. (2017). Rapid Maxillary Expansion and Upper Airway Morphology: A Systematic Review on the Role of Cone Beam Computed Tomography. BioMed research international, 2017 , 5460429. https://doi.org/10.1155/2017/5460429

Gogna, N., Johal, A. S., \& Sharma, P. K. (2020). A estabilidade da expansão maxilar rápida assistida cirurgicamente (SARME): Uma revisão sistemática. Revista de cirurgia cranio-maxilo-facial: publicação oficial da Associação Europeia para Cirurgia Cranio-Maxillo-Facial, 48(9), 845-852. https://doi.org/10.1016/j.jcms.2020.07.003

Kee-Joon, L., Sung-Hwan, C., Tae-Hyun, C., Kyung-Keun, S., \& Byeong-Tak, K. (2018). Maxillary transverse expansion in adults: Rationale, appliance design, and treatment outcomes. Seminars in Orthodontics. 24(1) 52-65. https://doi.org/10.1053/j.sodo.2018.01.006

Koudstaal, M. J., Poort, L. J., van der Wal, K. G., Wolvius, E. B., Prahl-Andersen, B., \& Schulten, A. J. (2005). Surgically assisted rapid maxillary expansion (SARME): a review of the literature. International journal of oral and maxillofacial surgery, 34(7), 709-714. https://doi.org/10.1016/j.ijom.2005.04.025

Kunz, F., Linz, C., Baunach, G., Böhm, H., \& Meyer-Marcotty, P. (2016). Expansion patterns in surgically assisted rapid maxillary expansion: Transpalatal distractor versus hyrax appliance. Expansionsmodus der chirurgisch unterstützten Gaumennahterweiterung: Transversaler Distraktor vs. Hyrax-Apparatur. Journal of orofacial orthopedics = Fortschritte der Kieferorthopadie: Organ/official journal Deutsche Gesellschaft fur Kieferorthopadie. 77(5), 357-365. https://doi.org/10.1007/s00056-016-0043-3

Lagravere, M. O., Major, P. W., \& Flores-Mir, C. (2006). Dental and skeletal changes following surgically assisted rapid maxillary expansion. International journal of oral and maxillofacial surgery, 35(6), 481-487. 
Research, Society and Development, v. 10, n. 4, e57910414357, 2021

(CC BY 4.0) | ISSN 2525-3409 | DOI: http://dx.doi.org/10.33448/rsd-v10i4.14357

Lehman Jr, J. A., \& Haas, A. J. (1989). Surgical-orthodontic correction of transverse maxillary deficiency. Clinics in plastic surgery, $16(4), 749-755$.

Lima, A. N. D., Detoni, E., Milani, B. D. A., Morando, F. S., \& Jorge, W. A. (2011). With bone anchorage device for surgically assisted maxillary expansion: a case report. Rev. cir. traumatol. buco-maxilo fac.11(4). https://doi.org/10.1590/S0034-72992006000400005

Neto, A. D. A., Sampaio, T. R. C., Santos, D. L. P., Filho, L. L. T. N., Filho, J. R. L., \& Nogueira, P. T. B. C. (2015). Rapid expansion surgically assisted maxillary with the use of bone-anchored distractor: Case report. BJSCR.11(1) 24-27. https://www.mastereditora.com.br/periodico/20150601_090128.pdf.

Neyt, N. M., Mommaerts, M. Y., Abeloos, J. V., De Clercq, C. A., \& Neyt, L. F. (2002). Problems, obstacles and complications with transpalatal distraction in non-congenital deformities. Journal of cranio-maxillo-facial surgery: official publication of the European Association for Cranio-Maxillo-Facial Surgery. 30(3), 139-143. https://doi.org/10.1054/jcms.2002.0304

Pereira, M. D., Koga, A. F., Prado, G. P. R., \& Ferreira, L.M. (2012). Complications From Surgically Assisted Rapid Maxillary Expansion With HAAS and HYRAX Expanders. Journal of Craniofacial Surgery. 29(2) 275-278. 10.1097/SCS.0000000000004079

Sahoo, N. K., Issar, Y., \& Thakral, A. (2019). Mandibular Distraction Osteogenesis. The Journal of craniofacial surgery, 30(8), 743-746. https://doi.org/10.1097/SCS.0000000000005753

Santos, S. E., Gonçalves, G. M., Sato, F. R. L., Lopes, M. C. A., \& Moreira, R. W. F. (2012). Rotterdam palatal distractor: an option for surgically assisted rapid maxillary expansion." Rev. cir. traumatol. buco-maxilo-fac. 12(4). https://www.revistacirurgiabmf.com/2012/v12.n4/v12n4.3.pdf

Suri, L., \& Taneja, P. (2008). Surgically assisted rapid palatal expansion: a literature review. American journal of orthodontics and dentofacial orthopedics: official publication of the American Association of Orthodontists, its constituent societies, and the American Board of Orthodontics, 133(2), 290-302. https://doi.org/10.1016/j.ajodo.2007.01.021

Tehranchi, A., Ameli, N., Najirad, Z., \& Mirhashemi, F. S. (2013). Comparison of the skeletal and dental changes of tooth-borne vs. bone-borne expansion devices in surgically assisted rapid palatal expansion: A finite element study. Dental research journal. 10(6), 777-783

Ylikontiola, L. P., Sándor, G. K., \& Harila, V. (2015). Perpendicular serial maxillary distraction osteogenesis in cleft lip and palate patients. Annals of maxillofacial surgery, 5(2), 148-157. https://doi.org/10.4103/2231-0746.175778

Zhou, Y., Long, H., Ye, N., Xue, J., Yang, X., Liao, L., \& Lai, W. (2014). The effectiveness of non-surgical maxillary expansion: a meta-analysis. European journal of orthodontics, 36(2), 233-242. https://doi.org/10.1093/ejo/cjt044 